\title{
3D Finite Element Analysis of Flat and Bent Plates for Crack Detection using Tone Burst Eddy Current Thermography
}

\author{
M.N. Libin*, Krishnan Balasubramaniam*, B.W.Maxfield* and C.V. Krishnamurthy** \\ * Centre for Nondestructive Evaluation, Indian Institute of Technology Madras, Chennai, INDIA \\ libinmn@gmail.com, balas@iitm.ac.in, bruce@emat.com \\ **Department of Physics, Indian Institute of Technology Madras, Chennai, INDIA \\ cvkmind@gmail.com
}

\begin{abstract}
Tone Burst Eddy current Thermography (TBET) is a new hybrid, non-contacting, non-destructive evaluation method with a wide area of applications including aircraft. Aircraft can have structural cracks or other defects which can lead to failure. The safe life of structural members can be evaluated by determining the size and orientation of critical cracks. For understanding cracks, fundamental knowledge about the induced current density distribution in the component under test is required. Further, this information enables us to find the amount of heat produced at those locations and how it diffuses to the surface. This paper describes finite element analysis of flat and bend aluminum plates for crack detection using TBET. 3D simulation results have been achieved by using COMSOL multi-physics with AC/DC module and general heat transfer. Various parameters considered in this study are a) crack dimension, b) excitation time and c) plate bend angle. A comparison of results obtained by varying the above mentioned parameters is done. At crack edges, induced current is seen concentrated thus indicating a localized high heating in those areas relative to other regions. TBET method was found well suited for the detection of service induced cracks, usually caused by either fatigue or stress corrosion, with a high degree of sensitivity.
\end{abstract}

\section{Introduction}

Tone Burst Eddy current Thermography (TBET) technique is widely used in aircraft industries for crack and corrosion detection under paints, cracks around the riveted joints, edge cracks, in transport industries for rolling contact failures, which leads to the cracks formation in rail track heads, etc. TBET technique combines both Eddy Current testing(ECT) and Thermographic non-destructive evaluation (TNDT) techniques to provide a fast and efficient method for defect detection and characterization over a relatively wide area[1]. This technique uses induced eddy currents to heat the material being tested and defect detection is based on the changes of the induced eddy current flows revealed by the thermal visualization captured by an infrared(IR) camera. Thermographic data and thermograms can then be immediately assessed to provide an indication of major faults and the data can be further analyzed to provide quantitative information of defects inside the inspected sample.

ECT involves the application of a high frequency (typically $150-450 \mathrm{kHz}$ ) electromagnetic wave to the material under inspection. For TNDT a short thermal stimulation pulse lasting from a few milliseconds for high-conductivity material(such as metals) to a few seconds for low-conductivity specimens(such as plastics, graphite epoxy laminates) is used[5]. In ECT much importance is attached to the relationship between electromagnetic skin depth(which is the depth of penetration of induced eddy current on the surface) and the defect depth, but as the skin depth formula is only applicable to perfectly flat and flawless samples, this relationship can only provide a rough guide at best. Since in TNDT we are dealing with only the distribution of surface temperature to assess the structure or behavior of what is under the surface, even if the skin depth is lesser than the defect depth the heat produced on the surface of the sample transfers from the warm areas to the cooler ones by conduction, which effectively occurs by diffusion.

This study considers the capabilities of TBET for obtaining quantitative information about crack type damage in aluminum flat and bend plates. The investigation is implemented by simulating the transient thermal distribution for cracks around rivets, edges and bended regions, via finite element solver COMSOL multi-physics with AC/DC module and general heat transfer module. Various parameters considered in this study are a)excitation time, b) crack depth and c) plate bend angle. A comparison of results obtained by varying the above mentioned parameters is done. At crack edges, induced current is seen concentrated thus indicating a localized high heating in those areas relative to other regions. Driven by the requirement of quantitative NDE means of characterizing crack defects, a study has been made of the response of TBET to edge crack[2] and riveted joint crack. Numerical simulations are carried out for a bend plate having crack at the bended region and a comparison is made for various plate bend angles. The ability to acquire quantitative information about defect geometry, i.e. depth, curved regions and length, are important for the accurate 
description of a defect. In this work, features from the temperature distributions of TBET investigations were extracted to gain quantitative information about edge crack and riveted joint crack.

\section{Numerical simulation for TBET - results and analysis}

The Simulation of TBET technique requires a multi-physics electro-thermal approach involving, (a) an electromagnetic model for the eddy-current generation, (b) an electro-thermal model for the conversion of eddy-currents into heat, and (c) the heat transfer model for the heat conduction from the heating surface into the material[2,6,7]. A 3D Finite Element Modeling (FEM) approach has been selected so that the model can be later extended to more complex and arbitrary configurations. All models were developed using the Multiphysics COMSOL $®$ package version 3.5a.

\subsection{Theoretical background}

Induction heating is accomplished using an electromagnetic induction coil through which alternating current at a specific frequency is applied. Whenever this primary AC passes through an electromagnetic induction coil with a certain frequency of excitation, a varying magnetic field will generate around the coil. When this electromagnetic field is applied to a conductive material placed near, an electro-magnetic force will generate which induces a current to flow through it. This induced secondary current is called eddy current. The secondary eddy-currents produced in the sample due to this primary current by induction coil causes a rise in the near surface temperature of the sample due to Joule heating. The presence of defect will disturb the eddy-current density distribution around it and hence the surface temperature distribution. A rise/fall of few degrees is expected for TBET when a defect is present. In most of the earlier reported work, continuous $\mathrm{AC}$ was employed at a single frequency. However, the use of tone bursts (a fixed number of cycles) and the effect of the frequency of the AC are both explored in great detail in this paper. This technique, henceforth called Tone Burst Eddy-current Thermography (TBET) uses tone burst AC pulses to locally heat a conducting material and uses a thermal imaging IR camera to map the surface temperatures.

Solving the magnetic field propagation (Eq. [1]) simultaneously with the heat transfer (Eq. [5]) is the underlining equations which governs the whole simulated system. The governing equation pertaining to magnetic field propagation is given by,

$$
\vec{\nabla} \times \frac{1}{\mu}(\vec{\nabla} \times \vec{A})+(\sigma+j \omega \varepsilon) \vec{\nabla} V+\left(j \sigma \omega-\varepsilon \omega^{2}\right) \vec{A}=\vec{J}_{e}+\sigma\left(\vec{v}_{e} \times(\vec{\nabla} \times \vec{A})\right)
$$

where, $\vec{A}$-Magnetic Vector Potential, $\vec{J}_{e}$-Eddy-current density, $\sigma-$

Electrical conductivity, $\omega-$ Circular frequency, $\vee$-Electric potential, $\varepsilon$-Electrical permeability, $\mu$-Magnetic permeability, $\vec{v}_{e}$-Velocity of the sample. The boundary conditions to solve the above equation are,

$$
\text { For all the interior boundaries, } \hat{n} \cdot\left(\vec{H}_{1}-\vec{H}_{2}\right)=0
$$

For the end boundary $\vec{A}=0$

The eddy-currents generated by induction due to Joule heating increase the average temperature of the body. The Joule losses are given by

$$
Q=\frac{\vec{J} \times \vec{J}^{*}}{2 \sigma}
$$

Where, $\vec{J}$ is the eddy-current in the sample, $\vec{J}^{*}$ is the complex conjugate of $\vec{J}$ and $\sigma$ is the electrical conductivity of the sample.

The Fourier's transient heat conduction equation for homogeneous isotropic material is given by

$$
\vec{\nabla}^{2} T+\frac{Q}{k}=\frac{1}{\alpha} \frac{\partial T}{\partial t}
$$

where, $\mathrm{T}$ is the temperature, $\alpha$ is the thermal diffusivity of the material, and $\mathrm{Q}$ is the heat source or heat sink. For the TBET simulations, the heat generated by Joule losses was incorporated into the above equation with appropriate boundary conditions to solve for the thermal profile at any arbitrary point in the solution domain as a function of time. Boundary conditions of convection and radiation are applied on both the sample and the coil through the following equation,

$$
-k \frac{\partial T}{\partial n}=h\left(T-T_{\infty}\right)+\varepsilon \sigma\left(T^{4}-T_{\infty}^{4}\right)+Q_{s}
$$

Where $\mathrm{T}_{\infty}=$ Ambient temperature, $\mathrm{T}$ is the temperature of the body, $\mathrm{h}$ is the convection coefficient, $\varepsilon$ is the emissivity of the body, $\sigma$ is the Stefan's constant for radiation, Qs is the surface source/sink of heat. 
Air domain boundary is considered as the constant temperature boundary condition with temperature equivalent to room temperature i.e.,

$\mathrm{T}=$ To, where To is equal to 300 Kelvin.

\subsection{Simulation model description}

Various parameters considered in simulation study are a)excitation time, b) crack length and c) plate bend angle. For the study of first two parameters two types of cracks are considered in the simulation, riveted joint crack and zdge crack. Schematic model used for the simulation of aluminum plate with riveted joint crack and edge crack are shown in Fig. 1(a) and 1(b) respectively. A riveted hole of $3 \mathrm{~mm}$ dia. with $5 \mathrm{~mm}$ crack length were modeled and the effect of these parameters on the thermal profile/image by varying one of them for a particular crack orientation were studied and compared. An aluminum plate of $150 * 100 * \mathrm{~d} \mathrm{~mm}^{3}$, where various plate thickness(d) considered are $1 \mathrm{~mm}, 2 \mathrm{~mm}, 3 \mathrm{~mm}$ and $3.5 \mathrm{~mm}$. The entire sub-domains are surrounded by an air domain of dimension $250 * 220 * 150 \mathrm{~mm}^{3}$. A constant lift-off distance of $5 \mathrm{~mm}$ was used for the inductor. For Edge crack modeling, an aluminum plate of $220 * 50 * 3 \mathrm{~mm}^{3}$ and a hollow copper induction inductor of outer radius $2.4 \mathrm{~mm}$, inner radius $1.9 \mathrm{~mm}$ and length $12 \mathrm{~mm}$ was used. Lift off used was $5 \mathrm{~mm}$. An aluminum plate of $220^{\star} 150 * 3 \mathrm{~mm}^{3}$ with bent angles $180^{\circ}, 120^{\circ}, 90^{\circ}$ and $60^{\circ}$ with the same dimension of inductor, air domain and lift off (as that of edge crack modeling) were used to study the effect of bend angle in the distribution of heat around crack tip. A full crack of dimension $5 \mathrm{~mm} \star 3 \mathrm{~mm} \star 0.05 \mathrm{~mm}$ is made at the center portion of bend plate. Fig. 2 shows the schematic of aluminum bend plate with center crack used for simulation.

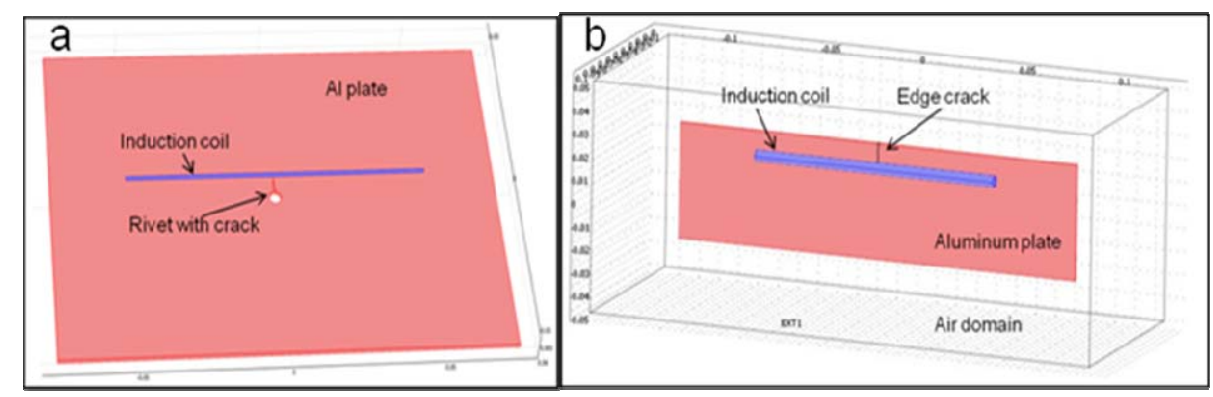

Figure 1. FEM model of the circular induction wire on an Aluminum sample that was used in the simulation havina (a). Riveted ioint crack (b) Edae crack

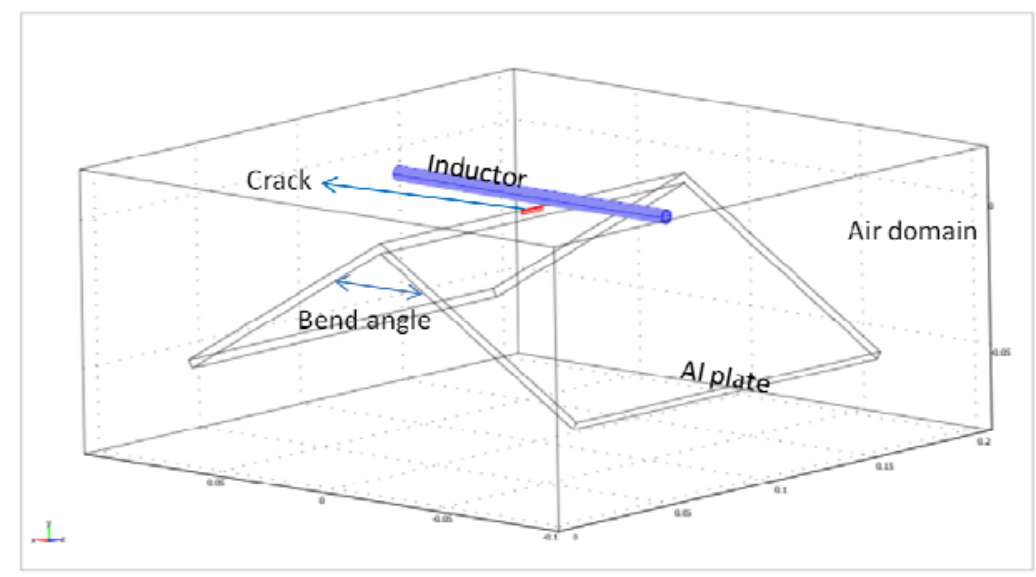

Figure 2. FEM model of the circular induction wire over a bend aluminum plate used for simulation

\subsection{Simulation results and analysis}

Numerical simulation results obtained for riveted joint crack by varying the parameters; excitation time and crack length were studied (Fig. 3). It is observed a higher heating around the crack tip and below surface region of riveted hole. Higher thermal contrast at the crack tip is because of the increased current density concentration at the crack tip. More over high current density distribution contributes a greater Joule heating results in high heat generation at those regions. Thermal contrast at the crack tip is visible more clearly at lower excitation times(10-50milli sec) and is observed 
distributing around it as the excitation time proceeds Fig. 3.a. As the plate thickness increases, temperature contrast is seen reducing at the transmission side for a constant lift off Fig. 3.b.

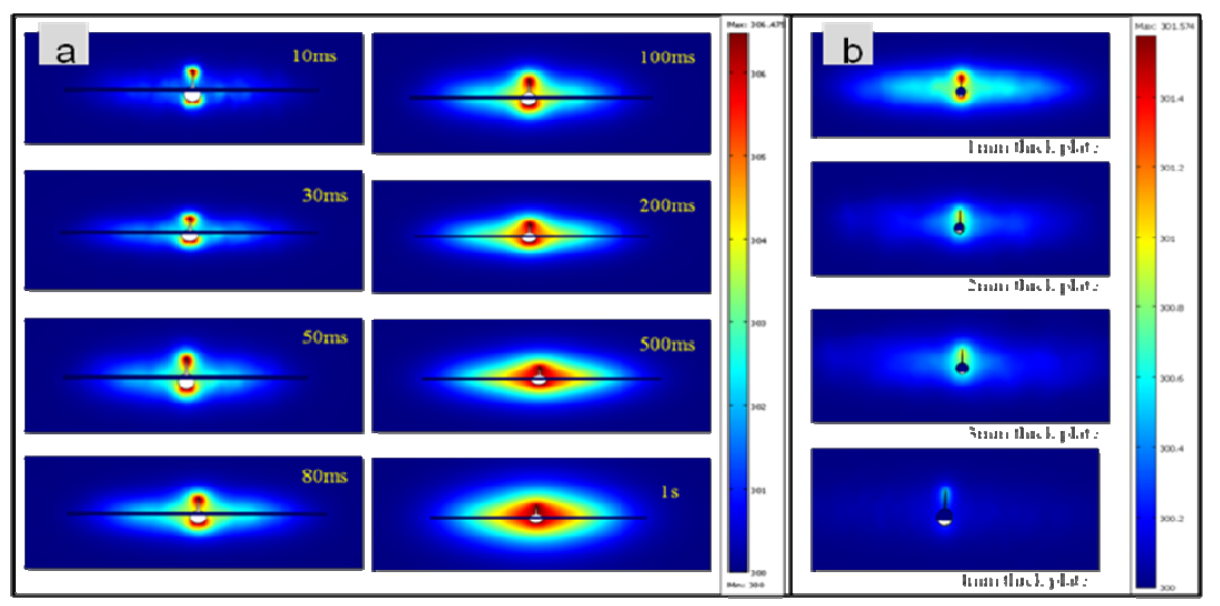

Figure 3. Comparison of thermal profile obtained for Aluminum plate having riveted joint crack for (a). Various time of excitation (b). Different plate thickness

For edge crack, thermal profile obtained at the crack tip is plotted for different excitation time (Fig. 8). A higher temperature contrast at the crack tip is clearly visible at lower excitation time (50milli sec) and as the excitation time passes heat flux is shown spreading around the crack tip.

Fig. 4.1 shows the simulation results obtained for bent aluminum plates both in transmission and reflection mode. Here also a high contrast of heating was observed around crack tip at lower excitation and the heat spreads around the tip as time proceeds. It is observed a rise in temperature at the crack tip at reflection side as the bend angle increases Fig. 4.2. The hike in the temperature with bend angle is due to the linear variation of the lift-off distance of plate with respect to inductor. Figure 5 shows thermal images of aluminum plate having three different crack length $(2.5 \mathrm{~mm}, 5 \mathrm{~mm}$, and $10 \mathrm{~mm})$ at reflection mode and transmission mode. It is observed a high localized heating around the cracks and is well observed at lower excitation time.

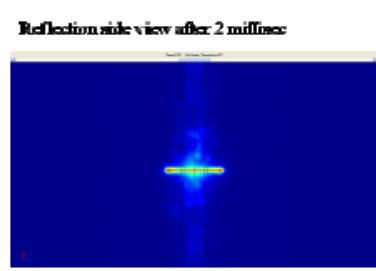

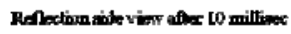
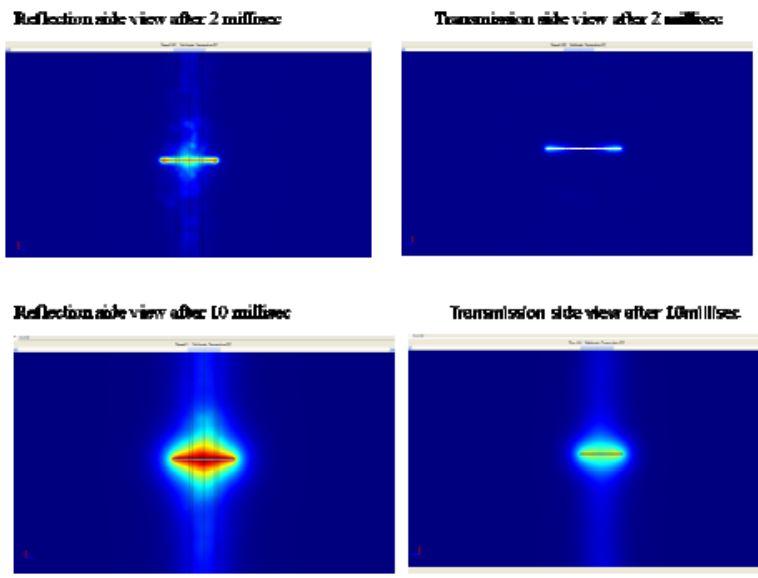

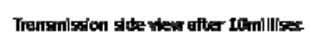
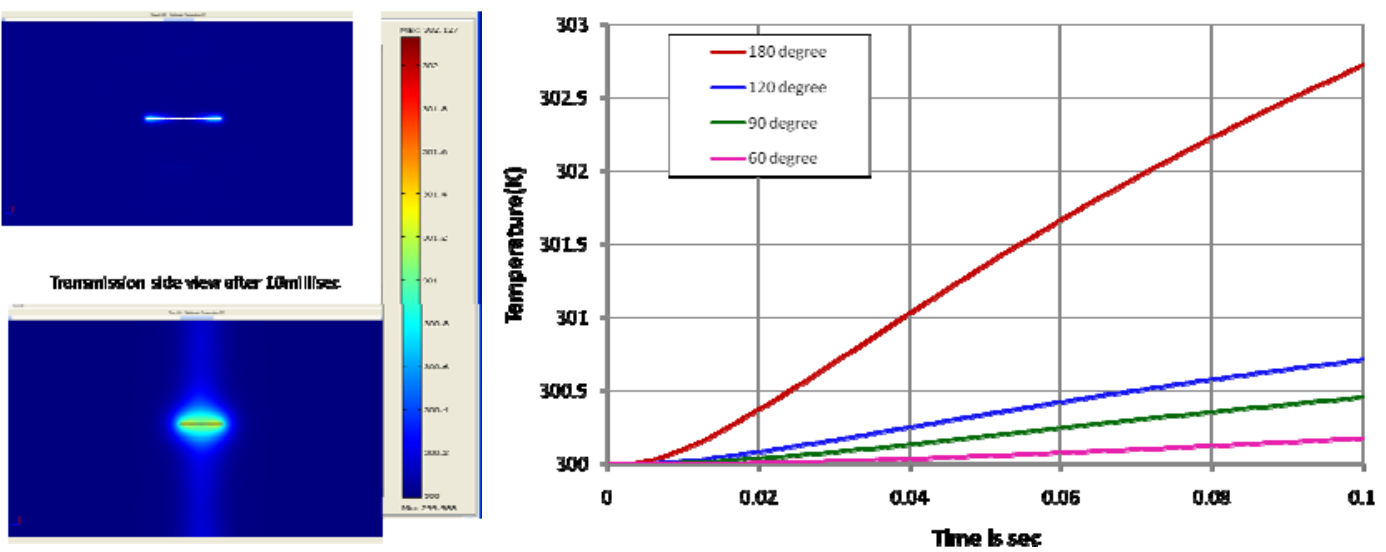

Figure 4. (1)Thermal image of 120 degree bend plate at reflection and transmission side (2) Thermal profile obtained for different bent angles 


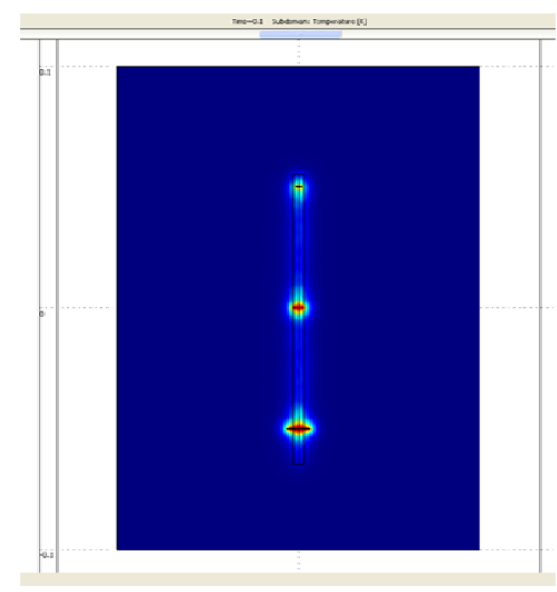

Reflection side
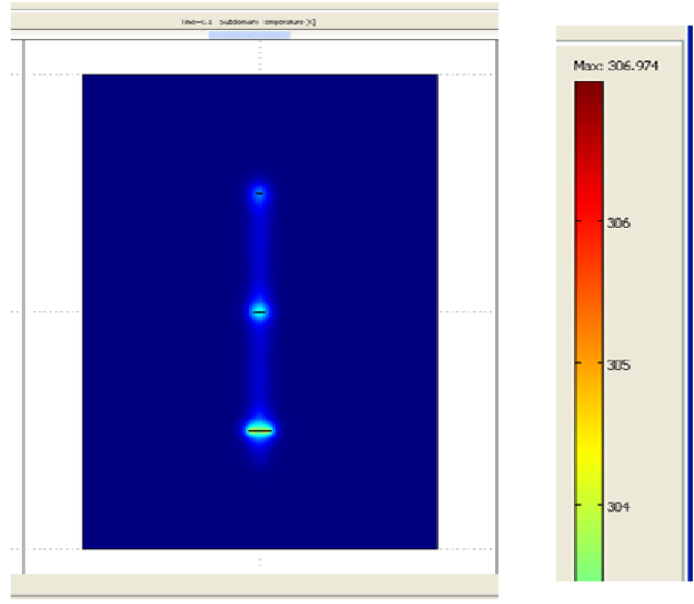

Transmission side

Figure 5. Thermal image at Reflection side and Transmission side after 10millisec of heating

\section{Experimental Validation}

Figure(6) shows the experiment set up for TBET. It consists of an induction heating control unit which supplies power to the work station(head). The work station contains a transformer coupled resonant circuit, including two capacitors and an excitation coil. Here the excitation of the coil is controlled by the function generator which also controls the excitation time for heating of the experiment. The excitation frequency is dictated by the values of the capacitors, the inductance of the coil and the load of the coil.

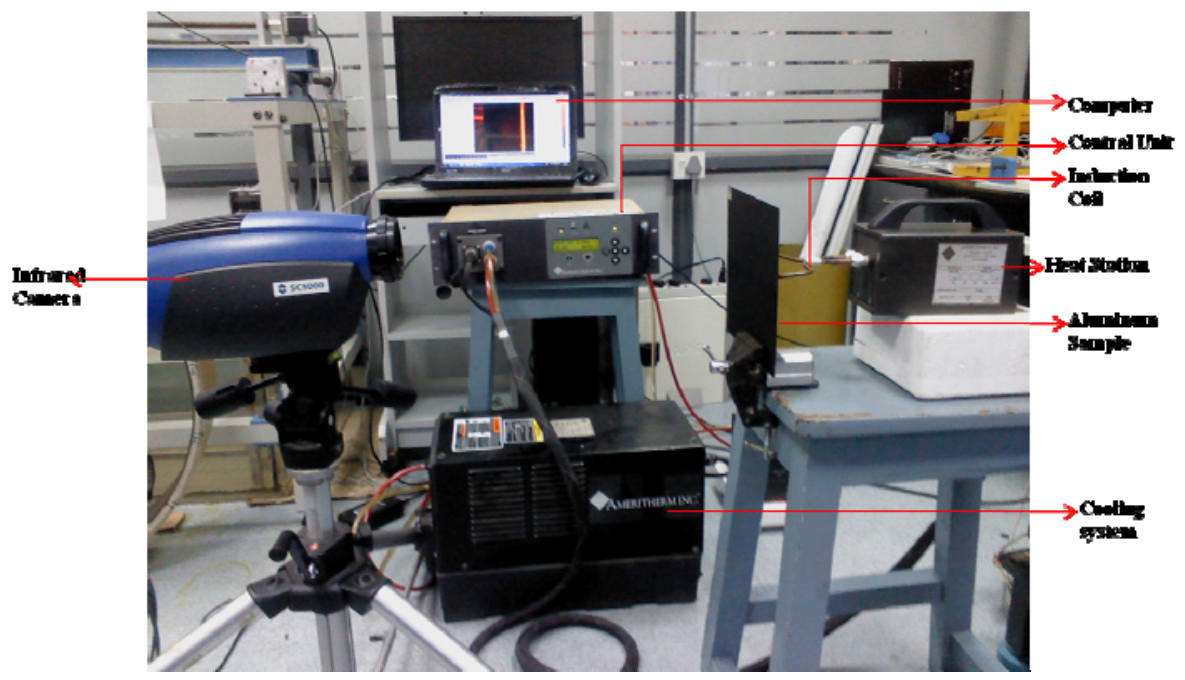

Figure 6. Experimental setup

The IR camera connected to the PC is used to capture thermal images. IR camera used for capturing thermal images was FLIR made SC5000 MWIR camera system and the AMERITHERM induction heating system was used for excitation, which has a maximum excitation power of $1 \mathrm{~kW}$, a maximum current of 1500A(RMS value) and an excitation frequency range $150-400 \mathrm{kHz}$. A circular copper coil was positioned normal to the test sample, with the edge of the coil perpendicular to the defect under inspection. For experiment a current of 800A RMS was applied to the induction coil for an excitation time of 100ms heating period (same value used in simulation also). The excitation frequency is determined by the resonant frequency of the circuit formed by the coil, the sample and the passive components 
contained in the work head of the AMERITHERM system. In the test the frequency is settled at $230 \mathrm{kHz}$. Thermal images are recorded at a frame rate of 380 frames/second and the acquired image sequences were processed in Altair.

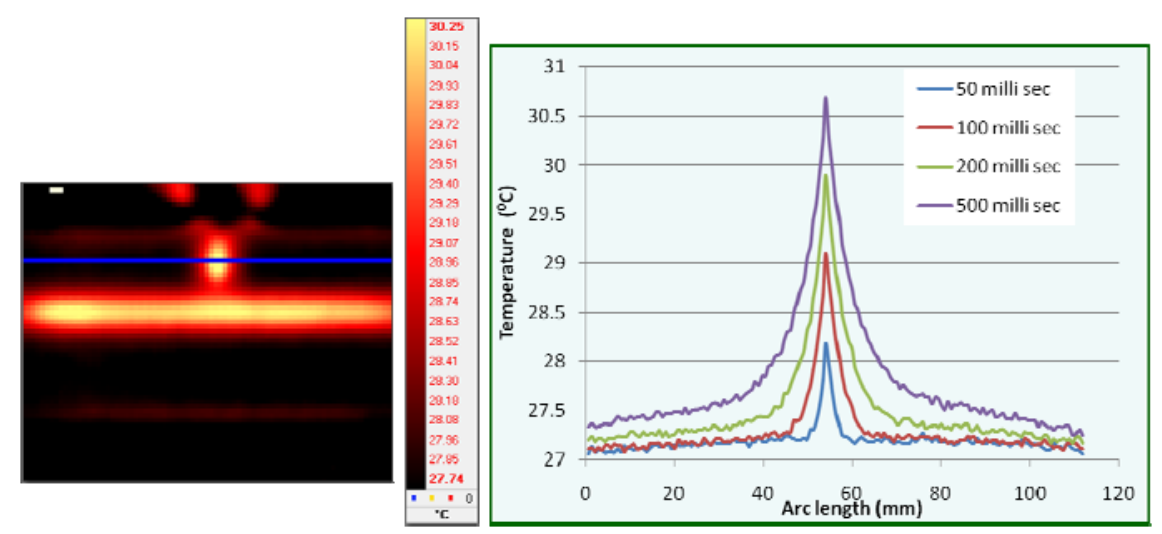

Figure 7 Experimental result of flat plate having edge crack

The Sample used for the experiment, as in the simulation work was a 2series aluminum sample having an edge crack of $5 \mathrm{~mm}$ length. Fig. 7 shows the experimental thermal image on the surface in hotmetal scale after 100ms of heating. In order to examine the temperature profile of the edge crack and to compare it with the simulation results, a temperature line profile at the sample surface near the induction coil was acquired for different interval of time, which is shown in the Fig.7. Fig. 8 shows the simulated thermal image and profile which is in good agreement with experimental results. Both the experiment and simulation temperature profile shows a high heating indicating the concentration of eddy current density around crack tip. Both the results implies a good temperature contrast at lower excitation time and the heat spreads and gets normalized around the crack tip as time proceeds.
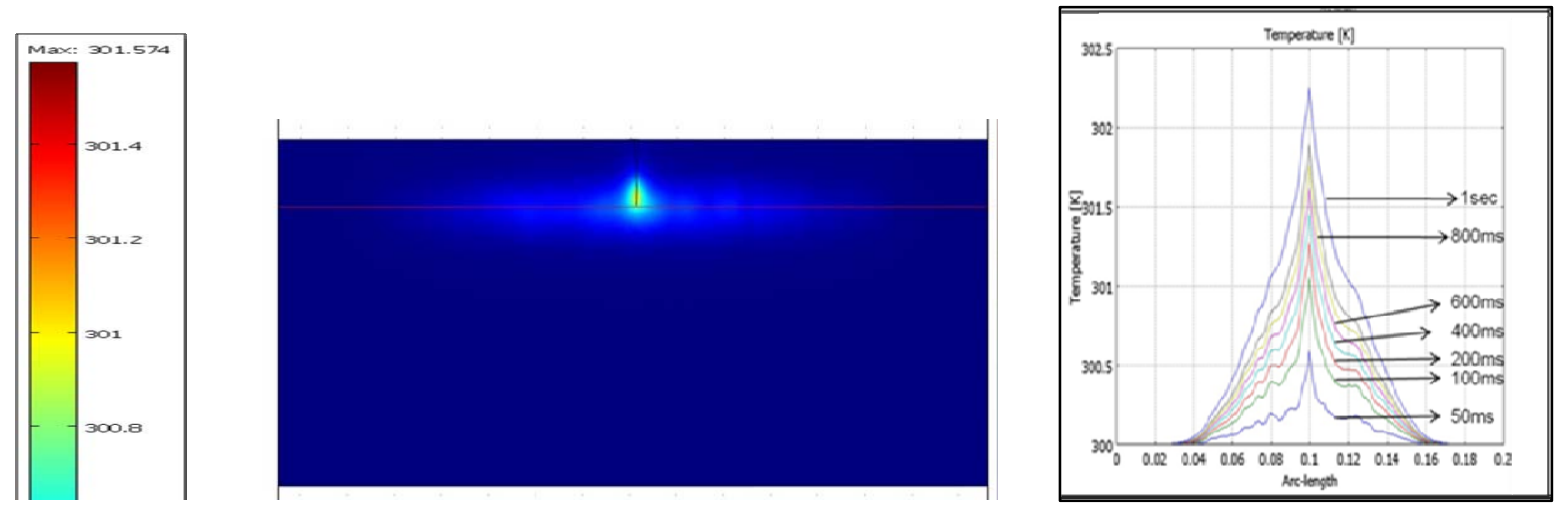

Figure 8 Experimental result of flat plate having edge crack 


\section{Conclusions}

An NDT technique, TBET was found useful technique for characterizing and quantifying cracks around the edges of plates and rivets, which are found common in aircraft components. Simulations were carried out for a particular dimension crack made at plate edge side and circular riveted hole side and the results were studied and compared for various parameters; crack length, time of excitation and plate bend angle. Current density is found concentrated around the crack tip and circular rivet sides that results in the high joule heating around those areas and can be well seen as a high thermal contrast regions in thermal profiles. Thermal contrast is well observed at lower excitation time for a particular frequency (typically of few $\mathrm{kHz}$ ) and heat flux will get distributed around the crack tip as excitation time proceeds. Also as the crack depth increases the thermal contrast at the transmission side is seen decreases for a constant lift off. Temperature is seen increasing as the plate bend angle increases both $\mathrm{R}$ and $\mathrm{T}$ mode respectively. The hike in the temperature near crack tip due to increase in the bend angle is due to the linear variation of the lift-off distance of plate with respect to the inductor.

\section{REFERENCES}

[1] Kiran Kumar N, Krishnamurthy CV, Maxfield BW, Krishnan Balasubramaniam. Tone Burst Eddy-current Thermography (TBET), Review of Progress in Quantitative Non Destructive Evaluation, 2007; Volume 27A:544551.

[2] Vrana J, Goldammer M, Baumann J, Rothenfusser M, Arnold W. Mechanisms smd models for crack detection with induction thermography, Review of Progress in QNDE, 2007; Vol 975: 475-482.

[3] Ilham Zainal Abidin, Gui Yun Tian, John Wilson, Suixian Yang, Darryl Almond, Quantitative evaluation of angular defects by Pulsed Eddy Current Thermography, NDT\&E International 43(2010) 537-546 .

[4] N. Biju, N. Ganesan, C.V. Krishnamurthy, Krishnan Balasubramaniam, Frequency Optimization for EddyCurrent, NDT \& E International, Volume 42, Issue 5, July 2009, Pages 415-420.

[5] Xavier P.V. Maldague, Theory and Practice of Infrared Technology for Nondestructive Testing, Chapter 10, 369-370, Wiley-Interscience, USA., 2001.

[6] Nathan Ida, Numerical Modeling for Electromagnetic Non-Destructive evaluation, Chapman \& Hill, ISBN 0412 468301.

[7] Maldague X. , 2001, "Infrared and thermal testing " Nondestructive testing Handbook, 3, ASNT Publications, Columbus, OH, USA.

[8] Stanley Zinn and S.L. Semiatin, Coil design and fabrication: basic design and modifications, Ameritherm, Inc., ASM International, (516) 338-5151. 